\title{
Effect of charge transfer on electrostatic adhesive force under different conditions of particle charge and external electric field
}

$\operatorname{AUTHOR}(S)$ :

Techaumnat, Boonchai; Matsusaka, Shuji

\section{CITATION:}

Techaumnat, Boonchai ... [et al]. Effect of charge transfer on electrostatic adhesive force under different conditions of particle charge and external electric field. Powder

Technology 2016, 301: 153-159

ISSUE DATE:

2016-11-01

URL:

http://hdl.handle.net/2433/229115

\section{RIGHT:}

(c) 2016. This manuscript version is made available under the CC-BY-NC-ND 40 license

http://creativecommons.org/licenses/by-nc-nd/4.0/; The full-text file will be made open to the public on 01 November 2018 in accordance with publisher's 'Terms and Conditions for Self-Archiving'; This is not the published version. Please cite only the published version.; この論文は出版社版でありません。引用の際には出版社版をご確認ご利用ください。 
Effect of Charge Transfer on Electrostatic Adhesive Force under Different Conditions of Particle Charge and External Electric Field

Boonchai Techaumnat ${ }^{1 *}$ and Shuji Matsusaka ${ }^{2}$

${ }^{1}$ Department of Electrical Engineering, Faculty of Engineering, Chulalongkorn University, Bangkok 10330, Thailand

${ }^{2}$ Department of Chemical Engineering, Kyoto University, Kyoto 615-8510, Japan

*Corresponding author:

Boonchai Techaumnat

boonchai.t@chula.ac.th

Tel. +662-218-6553, Fax. +662-218-6555 


\begin{abstract}
The electrostatics of charged particles are utilized for various applications. This paper presents an analysis of the electric field and electrostatic adhesive force on a charged dielectric particle lying on a conducting plane under an externally applied electric field. The purpose of the analysis is to quantitatively investigate the force variation when there is charge transfer between the particle and the conducting plane. We treat the distribution of charges as either uniform on the particle or partially on the lower half. The transferred charge density is assumed to be dependent on the applied electric field. The results show that the electric field is very strong near the contact point, where the charge transfer may occur. Without the charge transfer, the electrostatic adhesive force on a negatively charged particle increases when the applied field is in the upward direction from the plane. However, in the presence of charge transfer, the force may vary only slightly with the applied field or even show a reverse tendency if the transfer charge density depends significantly on the applied field.
\end{abstract}

Keywords: electric field, charged particle, electrostatic force, charge transfer, numerical field calculation 


\section{Introduction}

The electrostatics of particles are used in a variety of powder-related industry applications, such as electrostatic precipitators, painting, coating and separation [1]. In many cases, particles are charged and their movement is controlled by inserting an electric field. We sometimes need to transfer charged particles from one surface to another. For example, toner particles are detached from a photoconductor to a transfer belt or to a paper in electrophotography [2]. On the other hand, reentrainment of particles is regarded as a problematic issue in electrostatic precipitation [3]. The detachment of charged particles from a substrate involves the Coulomb force inserted by an electric field [4, 5], the electrical image force between the particle charges and their images with respect to the substrate [6-8] and other surface forces, such as the van der Waals force [9, 10]. For electrostatic applications related to particle manipulation such as the transfer of toner particles, the electrostatic force is predominant over the van der Waals force and the effect of the liquid bridge force is usually small by a hydrophobic treatment of particle surface.

Typically, charges are introduced on particles by triboelectricity [11-14], induction charging [15, 16] or corona discharge [17-19]. For dielectric particles, the condition of the equipotential does not hold on the particle surface. For this reason, the particles may have various forms of charge distribution on the surface, depending on the charging method and the amount of particle charges [20]. The charge distribution has an effect on the behavior of the electrostatic force acting on the particles. The electrostatic adhesion does not take place directly at the contact surface, but is a result from the longrange electrostatic image force acting on particle charge, which attracts a charged particle to the substrate. The electrostatic adhesive force increases remarkably if charges are nonuniformly concentrated near the contact point between a particle and a substrate [5, 21, 22]. Nonuniform charging can cause significant discrepancy between the measured adhesive force and the estimated one based on the point-charge model. The behavior of electrical force on a charged particle when subjected to an applied electric field also varies with the charge distribution on particle surface [5, 21, 23, 24].

Electrostatic adhesive force was recently investigated using air flow to detach dielectric particles from a conducting plane under an electric field where the particles were charged by tribocharging [25]. The results showed that the particles, which were negatively charged, exhibited a consistent increase in adhesion with increasing electric field in the downward direction. The opposite tendency was observed when the field was in the upward direction. These results contradict typical expectations because a downward electric field tends to lift a negatively charged particle from the substrate, thus reducing the adhesion. The effect of charge transfer between the particle and the conductor was suggested to be a possible cause of the aforementioned variation of the electrostatic adhesion in the experiments.

In this paper, we present an analysis of the electrostatic adhesive force between a charged dielectric particle and a conducting plane under an externally applied electric field. The charge is distributed either uniformly over the entire particle surface or partially on the lower half of the particle. A field-dependent charge transfer is assumed to occur on the particle surface near the conducting plane. The charge transfer may arise from the contact between different materials, as described in $[12,26,27]$. Nonlinear volume or surface resistivity of particle may also cause charge leakage. We apply a numerical field calculation to obtain the variation of the electrostatic force. The main objective of this work is to quantitatively study the effects of the charge transfer on the electrostatic adhesion under different conditions of particle charges and external field magnitudes.

\section{Configuration of Analysis}

Figure 1 shows the configuration used for the analysis in this work. A charged dielectric particle of radius $R$ lies on a grounded conducting plane under a uniform electric field $\mathbf{E}$ ext. The electric field is 
taken to be positive in the upward direction. We assume the dielectric constant $\varepsilon_{r}=3$ for the particle and $\varepsilon_{r}=1$ for the surrounding medium (air).

We consider two types of charge distribution on the particle before charge transfer occurs. Surface charge density $\sigma$ is equal to $\sigma_{0}$ and is uniform over the particle surface for the first type. For the second kind, the charge density is zero on the upper half and constant $\left(\sigma=\sigma_{0}\right)$ on the lower half of the particle surface, that is, the particle is partially charged in the latter case.

The change of the surface charge density due to charge transfer between the particle and the conducting plane is taken into account by designating Patch A that occupies $0 \leq \theta \leq \alpha$, where $\theta$ is the zenith angle measured from the contact point (See Fig. 1.). We assume that the charge density $\sigma_{A}$ on Patch A follows a relationship

$$
\sigma_{A}=\sigma_{0}+\sigma_{E 0}+k_{E} E_{e x t}
$$

where $\sigma_{E 0}$ is the transferred charge density in the absence of the externally applied electric field $E_{\text {ext }}$ and $k_{E}$ is a coefficient representing the effect of the field $E_{\text {ext }}$ on the charge transfer. This concept results from the previous studies $[26,28,29]$.

In the calculation, the particle radius $R$ is equal to $2.5 \mu \mathrm{m}$. We consider the original charge density $\sigma_{0}=-10,-20$ and $-30 \mu \mathrm{C} / \mathrm{m}^{2}$. The applied field $E_{\text {ext }}$ is between -3 and $3 \mathrm{kV} / \mathrm{c} \mathrm{m}$. These values are based on the recently reported experiment [25], where corrections are needed. That is, the actual unit of electric field is $\mathrm{kV} / \mathrm{m}$ (not V/m) for Figs. 11 and 13 in [25]. The patch angle $\alpha=15^{\circ}$. For the charge transfer, we use $\sigma_{E 0}$ values between -10 and $-30 \mu \mathrm{C} / \mathrm{m}^{2}$ and $k_{E}$ equal to 10,20 or $30\left(\mu \mathrm{C} / \mathrm{m}^{2}\right)(\mathrm{kV} / \mathrm{cm})^{-1}$.

The model of charge transfer in the current work assumes a change in particle charge near the contact point, which is not limited to a specific physical mechanism of charge transfer. For example, in the case of negatively charged toner particles, the contact point of the particle has a negative charge caused by the charge transfer. The parameters in Eq. (1) basically depend on the material properties such as work function, surface conductivity, and the energy states of charges. In addition, the patch angle $\alpha$ also depends on several factors such as particle geometry, field distribution, and the surface conductivity. However, it is still difficult to accurately estimate the values of $\sigma_{E 0}$ and $k_{E}$. In this work, we try to investigate how the values can affect the electrostatic force under the application of external electric field. First, we assume that the dielectric particle acquires negative charge by contacting with conducting plane; thus, the value of $\sigma_{E 0}$ arising from charge transfer is negative. The positive field in the upward direction should hinder the transfer of negative charge from the plane to the particle, and positive $k_{E}$ is then chosen here. The surface charge density is supposed to be the order of $10^{2} \mu \mathrm{C} / \mathrm{m}^{2}$ taking into account gas discharge of fine particles with surface roughness. In this condition, the absolute values of $\sigma_{E 0}$ and $k_{E}$ are tentatively assigned to the model.

Particle deformation can occur when a particle makes a contact with a substrate. The deformation has an effect on the contact area, and hence the charge transfer. However, under the application of strong electric field, the electric field alters significantly near the contact point. We expect that the influence of the electric field takes place on a wider area than the contact area, and the charge distribution based on the surface conductivity is more significant. Therefore, the contribution from the deformation may be negligible in this study.

\section{Calculation Method}

We apply the boundary element method [30], which is a numerical field calculation method, to determine the electric field distribution in the configuration of Fig. 1. The method is based on an integral 
relationship between the potential $\phi$ and the normal component $E_{n}$ of the electric field on the boundary of a domain. For potential $\phi_{i}$ at point $i$ in domain $\Omega$ enclosed by boundary $\Gamma$,

$$
C_{i} \phi_{i}=\int_{\Gamma} \psi\left(\mathbf{r}, \mathbf{r}_{\Gamma}\right) E_{n} \mathrm{~d} \Gamma+\int_{\Gamma} \frac{\partial \psi\left(\mathbf{r}, \mathbf{r}_{\Gamma}\right)}{\partial n} \phi \mathrm{d} \Gamma
$$

In the equation, $\mathbf{r}$ is the position of $i, \mathbf{r}_{\Gamma}$ is the position on boundary $\Gamma, \psi$ is the fundamental solution, and $C_{i}$ is a constant. For a smooth boundary $\Gamma, C_{i}=1 / 2$ if $i$ is on $\Gamma$ and $C_{i}=1$ if $i$ is in $\Omega$ but not on $\Gamma$. For the configuration and the charging condition considered in this work, the potential is axisymmetric about the $z$ axis in Fig. 1. The calculation is carried out using the axisymmetric coordinates shown in the figure. That is, $\mathbf{r}$ is defined by $(\rho, z)$ coordinates and $\mathbf{r}_{\Gamma}$ by $\left(\rho_{\Gamma}, z_{\Gamma}\right)$. Note that $E_{n}$ is taken to be positive in the direction outward from $\Omega$. The fundamental solution is expressed as

$$
\psi\left(\mathbf{r}, \mathbf{r}_{\Gamma}\right)=\frac{K(\sqrt{2 n /(m+n)})}{\sqrt{m+n}}
$$

where $K$ is the complete elliptic integral of the first kind, and

$$
\begin{aligned}
& m=\rho^{2}+\rho_{\Gamma}^{2}+\left(z-z_{\Gamma}\right)^{2} \\
& n=2 \rho \rho_{\Gamma}
\end{aligned}
$$

For the interior of the particle, Eq. (2) becomes

$$
C_{i} \phi_{i}=\int_{S} \psi\left(\mathbf{r}, \mathbf{r}_{\Gamma}\right) E_{n}^{I} \mathrm{~d} \Gamma+\int_{S} \frac{\partial \psi\left(\mathbf{r}, \mathbf{r}_{\Gamma}\right)}{\partial n} \phi \mathrm{d} \Gamma
$$

where $S$ is the particle surface, and the superscript $I$ denotes the field component inside the particle. For the exterior of the particle, we take the normal component $E_{n}^{E}$ in the same direction as $E_{n}^{I}$ and modify Eq. (2) to include the contribution of $\mathbf{E}_{e x t}$ as

$$
C_{i} \phi_{i}=-\int_{S} \psi\left(\mathbf{r}, \mathbf{r}_{\Gamma}\right) E_{n}^{E} \mathrm{~d} \Gamma+\int_{S} \frac{\partial \psi\left(\mathbf{r}, \mathbf{r}_{\Gamma}\right)}{\partial n} \phi \mathrm{d} \Gamma-\mathbf{E}_{e x t} \cdot\left(\mathbf{r}-\mathbf{r}_{0}\right)
$$

The superscript $E$ denotes the normal field component on $S$ in the exterior of the particle, and $\mathbf{r}_{0}$ is the position of the reference potential. The relationship between the normal electric field on both sides of the particle surface $S$ can be written as

$$
\varepsilon_{0}\left(E_{n}^{E}-\varepsilon_{r} E_{n}^{I}\right)=\sigma
$$

For the boundary element method, the contour of the particle is discretized into elements. The potential $\phi$ and normal electric field $E_{n}$ are interpolated as a function of the corresponding nodal values on each element. The existence of the conducting plane and the induced charges on the plane is taken into account by using image elements of the particle surface below the conducting plane to fulfill the condition of constant potential. Eqs. (6)-(8) are applied to each node position to construct a linear equation system, which is solved for the nodal $\phi$ and $E_{n}$ values on the particle. An inhouse program is used for the calculation. Approximately 1440 second-order curved elements are utilized for the particle contour to attain high accuracy of the calculation results. After obtaining the field solution, we determine the electrostatic force $\mathbf{F}$ acting on the particle by integrating the Maxwell stress over particle surface $S$,

$$
\mathbf{F}=\varepsilon_{0} \int_{S} \mathbf{E} E_{n}^{E}-\frac{1}{2} E^{2} \mathbf{n} \mathrm{d} s
$$


where $\mathbf{n}$ is the unit normal vector on $S$. The electric fields are taken from the exterior side for the integration.

\section{Results and Discussion}

\subsection{Uniformly Charged Particle without Charge Transfer}

Fig. 2 shows the distribution of the normal component $E_{n}$ of the electric field (in the exterior) on the surface of a uniformly charged particle when the applied field does not exist. The angle $\theta$ is measured from the contact point. The electric field is very strong near the contact point where $\theta=0^{\circ}$. The calculated electric field is significantly higher than the typical dielectric strength of air. However, the field decreases rapidly with distance from the particle and cannot induce electrical discharge by the streamer mechanism. However, such a strong electric field in Fig. 2 may lead to the transfer of charge between the particle and the conducting plane, which are very close to each other near the contact point.

Insertion of an external field $\mathbf{E}_{\text {ext }}$ may increase or decrease the electric field distribution near the contact point. Fig. 3 shows the variation of the normal electric field $E_{n}$ with applied field $\mathbf{E}_{\text {ext }}$ for a particle uniformly charged to $\sigma=-10 \mu \mathrm{C} / \mathrm{m}^{2}$. A positive electric field (i.e., in the upward direction) intensifies the electric field near the contact point but mitigates the field on the upper half of the particle. A negative field value has the opposite effect on the field distribution.

Figs. 4(a) and 4(b) show the distribution of the tangential electric field $E_{t}$ on the particle surface for a uniformly charged particle with $\sigma_{0}=-10$ and $-30 \mu \mathrm{C} / \mathrm{m}^{2}$, respectively. A positive tangential field is defined in the direction of increasing $\theta$, i.e., away from the contact point. We can see from the figure that $E_{t}$ is strongly positive near the contact point with the peak located around $\theta=15^{\circ}$. On this area, the Coulomb force due to positive $E_{t}$ acts on a negative charge in the $-\theta$ direction. Therefore, $E_{t}$ tends to move the particle charges to the vicinity of the contact point. The tangential field and the force due to $E_{t}$ are enhanced by the application of positive $E_{\text {ext }}$.

Comparison between Fig. 3 and Fig. 4(a) shows that the magnitudes of the tangential field are much smaller than those of the normal electric field. We may consider the roles of the normal and tangential field components as follows. The normal field $E_{n}$ makes a major contribution to the transfer of charge between the particle and the plane, whereas the tangential field indirectly assists the charge transfer by moving charges along the particle surface to the vicinity of the contact point. The movement of charges along a surface may require a lower electric field than that required for a direct detachment of charges from the surface.

Fig. 5 shows the electrostatic adhesive force $F_{a}$, the downward component of $\mathbf{F}$, on uniformly charged particles with different charge density $\sigma_{0}$ as a function of the applied field $E_{\text {ext. }}$. The electrostatic force becomes stronger with increasing field magnitude in the upward direction. The contribution of $E_{\text {ext }}$ to $F_{a}$ can be considered from the Coulomb force acting on the surface charges for the range of $E_{\text {ext }}$ in this figure because negative charges on the particle are attracted to the plane by an upward electric field. The $F_{a}-E_{e x t}$ relationships are approximately linear for the considered $\sigma_{0}$ and $E_{\text {ext }}$ values.

\subsection{Partially Charged Particle without Charge Transfer}

Fig. 6 shows the distribution of $E_{n}$ as a function of $\theta$ on a particle partially charged from $\theta=0$ to $90^{\circ}$ in the absence of an applied field. The field distribution is similar to that in Fig. 2 on the lower half, but the field is very small on the upper half due to the absence of surface charge. For the same $\sigma_{0}$ value, the total charge amount on the partially charged particle is equal to half of that on the uniform charge. 
Therefore, Fig. 6 and Fig. 2 imply that the electric field will be stronger in the case of partial charging if the total charge amounts are the same.

The effects of the external field $\mathbf{E}_{\text {ext }}$ on the normal electric field for the partially charged particle are not shown here, but they are similar to those for the uniformly charged particle. Fig. 7 shows the variation of the tangential field $E_{t}$ on the partially charged particle. On most of the charged area, $E_{t}$ still acts to move negative charges along the surface to the contact point. Near the edge of the charged area, the negative $E_{t}$ repulses charges to the upper half. An application of positive (upward) Eext increases the field value and reduces the area of negative $E_{t}$.

Fig. 8 shows the variation of the adhesive force $F_{a}$ with the magnitude $E_{\text {ext }}$ of the externally applied electric field on the partially charged particle. The force behavior is similar to that in Fig. 5 for the uniformly charged particle, that is, the adhesion increases with increasing upward electric field $E_{\text {ext }}$, and the $F_{a}-E_{e x t}$ relationships are approximately linear for the considered ranges of $\sigma_{0}$ and $E_{\text {ext. }}$. The electrostatic force varies linearly with the square of the electric field on the particle surface. Therefore, the adhesion is usually stronger on a partially charged particle than on a uniformly charged particle for the same total amount of charge and applied electric field. For example, in the absence of $E_{\text {ext }}, F_{a}$ magnitude is $0.61 \mathrm{nN}$ on the partially charged particle with $\sigma_{0}=-20 \mu \mathrm{C} / \mathrm{m}^{2}$, higher than $0.35 \mathrm{nN}$ on the uniformly charged particle with $\sigma_{0}=-10 \mu \mathrm{C} / \mathrm{m}^{2}$.

\subsection{Force Variation due to Charge Transfer}

The aforementioned results show that both uniform and partial charge distributions exhibit an increase of electrostatic adhesion when applying an upward electric field, and vice versa. The adhesive force behavior varies linearly with the applied field for the considered ranges of $\sigma_{0}$ and $E_{e x t}$. This section investigates the variation of the adhesive force behavior when there are charges transferred between the particle and the conducting plane. We consider that the charge transfer is dependent on the applied electric field $E_{\text {ext }}$, as given in Eq. (1). We assume a patch angle $\alpha=15^{\circ}$, which is approximately the angle of the peak tangential field before charge transfer occurs.

Figs. 9(a) and 9(b) show the calculation results of the uniformly charged particle when $\sigma_{E 0}=-10$ and $-30 \mu \mathrm{C} / \mathrm{m}^{2}$, respectively, and $k_{E}=20\left(\mu \mathrm{C} / \mathrm{m}^{2}\right)(\mathrm{kV} / \mathrm{cm})^{-1}$. The $F_{a}-E_{\text {ext }}$ characteristics are given on each graph for three values of the original charge density $\sigma_{0}$. Fig. 9 shows that the behavior of $F_{a}$ changes significantly from that in Fig. 5 for the uniformly charged particle. The adhesive force increases or decreases only slightly with the change in the applied electric field $E_{\text {ext }}$. Although the force magnitudes depend on the original charge density $\sigma_{0}$, the $F_{a}-E_{\text {ext }}$ characteristics are similar for the same $\sigma_{E 0}$ and $k_{E}$ values. A comparison between Figs. 9(a) and 9(b) shows that the larger $\sigma_{E 0}$ value results in stronger adhesive force. However, the effect of $\sigma_{E 0}$ is small for positive $E_{\text {ext }}$ values.

Fig. 10 shows the variation of $F_{a}$ with $E_{\text {ext }}$ on the uniformly charged particle when $\sigma_{E 0}=-30 \mu \mathrm{C} / \mathrm{m}^{2}$ and $k_{E}=10$ or $30\left(\mu \mathrm{C} / \mathrm{m}^{2}\right)(\mathrm{kV} / \mathrm{cm})^{-1}$ are used as the charge transfer parameters. For the smaller $k_{E}$ value in Fig. 10(a), the particle exhibits an increase of adhesive force with increasing applied field $E_{\text {ext }}$. The $F_{a}-E_{\text {ext }}$ characteristics in the figure are similar to the corresponding cases without charge transfer shown in Fig. 5.

With the higher $k_{E}$ value, the adhesive force in Fig. 10(b) is weakened with increasing electric field. From $E_{\text {ext }}=-3 \mathrm{kV} / \mathrm{cm}$, the decrease of the adhesive force is moderate but consistent. The force reaches its minimum at an $E_{\text {ext }}$ value between 2 and $3 \mathrm{kV} / \mathrm{cm}$. We may observe the roles of the charge transfer from the change in the normal electric field $E_{n}$, which makes a major contribution to the electrostatic force (as $E_{n}$ is much larger than $E_{t}$ ). Fig. 11 illustrates the variation of $E_{n}$ on the particle with $E_{\text {ext }}$ corresponding to the cases in Fig. $10($ b) $)$ i.e., $\sigma_{E 0}=-10 \mu \mathrm{C} / \mathrm{m}^{2}, \sigma_{E 0}=-30 \mu \mathrm{C} / \mathrm{m}^{2}$ and $k_{E}=30\left(\mu \mathrm{C} / \mathrm{m}^{2}\right)$ 
$(\mathrm{kV} / \mathrm{cm})^{-1}$. It is clear that the charge transfer reduces $E_{n}$ near the contact point with increasing $E_{\text {ext }}$ from -1 to $1 \mathrm{kV} / \mathrm{cm}$. The change of $E_{n}$ with $E_{\text {ext }}$ shows an opposite tendency in comparison with that in Fig. 3 . The reduction of $E_{n}$ weakens the electrostatic attractive force on the particle.

Note that the external field induces polarization in the dielectric particle. The interaction between the polarization charge and its image on the conducting plane yields an increase in the attractive electrostatic force. The effect of polarization becomes more important with increasing the external electric field. As a result, a further increase of $E_{\text {ext }}$ higher than $2-3 \mathrm{kV} / \mathrm{cm}$ results in stronger adhesive force in Fig. 10(b) due to the predominant attractive force on the polarization charge [31].

Figs. 12 and 13 show the variation of the adhesive force on the partially charged particle when charge transfer occurs. For $k_{E}=20\left(\mu \mathrm{C} / \mathrm{m}^{2}\right)(\mathrm{kV} / \mathrm{cm})^{-1}$, the adhesive force in Fig. 12 is reduced by increasing the electric field for both values of $\sigma_{E 0}$. The change in $\sigma_{E 0}$ has a more prominent effect on the adhesive force under a strong negative field than under a positive field. Comparing Fig. 12 with Fig. 9, we can see that the contribution of the charge transfer is more clearly seen on the partially charged particle.

Fig. 13 shows the $F_{a}-E_{\text {ext }}$ behavior for different $k_{E}$, which demonstrates that the $k_{E}$ value significantly affects the behavior of $F_{a}$. For the partially charged particle having $\sigma_{0}$ between -10 and $-30 \mu \mathrm{C} / \mathrm{m}^{2}$, the force minimally varies with the electric field when the smaller $k_{E}$ value is used in Fig. 13(a). On the other hand, with the larger $k_{E}$ value, there is a clear reduction of the adhesive force with increasing applied electric field. The adhesive force reaches its minimum when $E_{\text {ext }}$ is approximately $2-3 \mathrm{kV} / \mathrm{cm}$, similar to the cases of the uniformly charged particle.

In summary, our calculation results in this work demonstrate that charge transfer between the particle and the plane may have a significant role in the electrostatic adhesion of the particle if the parameter $k_{E}$, representing the dependency on the electric field, is sufficiently large. For the same $\sigma_{0}, \sigma_{E 0}$ and $k_{E}$ values, the effect of charge transfer is more prominent on the partially charged particle than on the uniformly charged particle. If charge is distributed on a smaller area of the particle surface, the charge transfer is expected to have a greater role in the adhesion, which causes the reduction of electrostatic adhesion with increasing electric field in the experiments.

\section{Conclusions}

In this work, we analyzed the effects on the electrostatic adhesion of the charge transfer between a charged dielectric particle and a conducting plane. In the absence of charge transfer, the adhesion of a negatively charged particle becomes stronger with an application of external electric field upward from the plane. However, a change in the surface in a small area near a contact point mitigates the effect of the applied field or even reverses the tendency of the force variation with the applied field, that is, the electrostatic adhesion may be weakened by the upward electric field, provided the degree of the charge transfer varies significantly with the magnitude of the external field.

\section{Acknowledgments}

BT wants to thank the Thailand Research Fund (TRF) for the support of this work.

\section{References}

[1] J.S. Chang, A.J. Kelly, J.M. Crowley, Handbook of Electrostatic Processes, CRC Press 1995.

[2] D.A. Hays, Paper documents via the electrostatic control of particles, Journal of Electrostatics, 5152 (2001) 57-63. 
[3] K. Parker, Electrical Operation of Electrostatic Precipitators, Institution of Engineering and Technology 2003.

[4] J.Q. Feng, D.A. Hays, A finite-element analysis of the electrostatic force on a uniformly charged dielectric sphere resting on a dielectric-coated electrode in a detaching electric field, Ieee Transactions on Industry Applications, 34 (1998) 84-91.

[5] J.Q. Feng, E.A. Eklund, D.A. Hays, Electric field detachment of a nonuniformly charged dielectric sphere on a dielectric coated electrode, Journal of Electrostatics, 40-1 (1997) 289-294.

[6] J.Q. Feng, Electrostatic interaction between two charged dielectric spheres in contact, Phys Rev E, 62 (2000) 2891-2897.

[7] M. Takeuchi, Adhesion forces of charged particles, Chem Eng Sci, 61 (2006) 2279-2289.

[8] T. Matsuyama, H. Yamamoto, The Electrostatic Force Between a Partially Charged Dielectric Particle and a Conducting Plane, Particle \& Particle Systems Characterization, 24 (2007) 79-84.

[9] J.N. Israelachvili, 6 - Van der Waals Forces, Intermolecular and Surface Forces (Third Edition), Academic Press, San Diego, 2011, pp. 107-132.

[10] J.Q. Feng, D.A. Hays, Relative importance of electrostatic forces on powder particles, Powder Technol, 135-136 (2003) 65-75.

[11] B.A. Kwetkus, Particle Triboelectrification and Its Use in The Electrostatic Separation Process, Particulate Science and Technology, 16 (1998) 55-68.

[12] S. Matsusaka, H. Maruyama, T. Matsuyama, M. Ghadiri, Triboelectric charging of powders: A review, Chem Eng Sci, 65 (2010) 5781-5807.

[13] A. Zarrebini, M. Ghadiri, M. Dyson, P. Kippax, F. McNeil-Watson, Tribo-electrification of powders due to dispersion, Powder Technol, 250 (2013) 75-83.

[14] K. Takagi, G.S.P. Castle, M. Takeuchi, Tribocharging mechanism of mono-component irregular and spherical toners in an electrophotographic development system, Powder Technol, 135-136 (2003) $35-42$.

[15] N. Toljic, G.S.P. Castle, K. Adamiak, Charge to radius dependency for conductive particles charged by induction, Journal of Electrostatics, 68 (2010) 57-63.

[16] Y. Wu, G.S.P. Castle, I.I. Inculet, S. Petigny, G. Swei, Induction charge on freely levitating particles, Powder Technol, 135-136 (2003) 59-64.

[17] M. Lackowski, K. Adamiak, A. Jaworek, A. Krupa, Electrostatic charging of particulates by ionic current in alternating electric field, Powder Technol, 135-136 (2003) 243-249.

[18] M. Jędrusik, A. Świerczok, The correlation between corona current distribution and collection of fine particles in a laboratory-scale electrostatic precipitator, Journal of Electrostatics.

[19] I. Panich, Corona discharge in a cylindrical triode charger for unipolar diffusion aerosol charging, Journal of Electrostatics, 70 (2012) 136-143.

[20] J.R. Melcher, Continuum Electromechanics, MIT Press 1981.

[21] B. Techaumnat, M. Kadonaga, Electrostatic force behavior of a nonuniformly charged particle on a planar dielectric solid, Dielectrics and Electrical Insulation, IEEE Transactions on, 18 (2011) 13661373.

[22] B. Techaumnat, T. Takuma, Analysis of the electrostatic force on a dielectric particle with partial charge distribution, Journal of Electrostatics, 67 (2009) 686-690.

[23] B. Techaumnat, M. Kadonaga, T. Takuma, Analysis of Electrostatic Adhesion and Detachment of a Nonuniformly Charged Particle on a Conducting Plane, Dielectrics and Electrical Insulation, IEEE Transactions on, 16 (2009) 704-709.

[24] B.A. Kemp, J.G. Whitney, Nonlinear nature of micro-particle detachment by an applied static field, Appl Phys Lett, 102 (2013) 141605.

[25] S. Matsusaka, D. Wei, M. Yasuda, S. Sasabe, Adhesive strength distribution of charged particles on metal substrate in external electric field, Advanced Powder Technology, 26 (2015) 149-155.

[26] S. Matsusaka, Control of Particle Tribocharging, KONA Powder and Particle Journal, 29 (2011) 27-38.

[27] G.S.P. Castle, Contact charging between insulators, Journal of Electrostatics, 40-1 (1997) 13-20.

[28] P. Bunchatheeravate, J. Curtis, Y. Fujii, S. Matsusaka, Prediction of particle charging in a dilute pneumatic conveying system, AIChE Journal, 59 (2013) 2308-2316. 
[29] M. Mizutani, M. Yasuda, S. Matsusaka, Advanced characterization of particles triboelectrically charged by a two-stage system with vibrations and external electric fields, Advanced Powder Technology, 26 (2015) 454-461.

[30] C.A. Brebbia, L.C. Wrobel, J.C.F. Telles, Boundary Element Techniques: Theory and Applications in Engineering, Springer-Verlag, Berlin 1984.

[31] T.B. Jones, Electromechanics of particles, Cambridge University Press 2005. 


\section{Figure Captions}

Fig. 1. Configuration of a charged dielectric particle under an externally applied field $\mathbf{E}_{\text {ext }}$.

Fig. 2. Distribution of the normal electric field on a uniformly charged particle in the absence of applied field $\mathbf{E}_{e x t}$.

Fig. 3. Variation with the applied field $\mathbf{E}_{\text {ext }}$ of the normal electric-field distribution on a particle uniformly charged to $-10 \mu \mathrm{C} / \mathrm{m}^{2}$.

Fig. 4. Tangential electric field on a uniformly charged particle with $\sigma_{0}$ equal to (a) -10 and (b) -30 $\mu \mathrm{C} / \mathrm{m}^{2}$.

Fig. 5. Adhesive electrostatic force $F_{a}$ as a function of $E_{\text {ext }}$ on a uniformly charged particle for different $\sigma_{0}$ values.

Fig. 6. Distribution of the normal electric field on a partially charged particle in the absence of applied field $\mathbf{E}_{\text {ext. }}$.

Fig. 7. Tangential electric field on a partially charged particle with $\sigma_{0}$ equal to (a) -10 and (b) -30 $\mu \mathrm{C} / \mathrm{m}^{2}$.

Fig. 8. Adhesive electrostatic force $F_{a}$ as a function of $E_{e x t}$ on a partially charged particle for different $\sigma_{0}$ values.

Fig. 9. Variation of $F_{a}$ with $E_{\text {ext }}$ on a uniformly charged particle in the presence of charge transfer with $k_{E}=20\left(\mu \mathrm{C} / \mathrm{m}^{2}\right)(\mathrm{kV} / \mathrm{cm})^{-1}$ and $\sigma_{E 0}=(\mathrm{a})-10$ and (b) $-30 \mu \mathrm{C} / \mathrm{m}^{2}$.

Fig. 10. Variation of $F_{a}$ with $E_{\text {ext }}$ on a uniformly charged particle with $\sigma_{E 0}=-30 \mu \mathrm{C} / \mathrm{m}^{2}$ and $k_{E}=(\mathrm{a})$ 10 or (b) $30\left(\mu \mathrm{C} / \mathrm{m}^{2}\right)(\mathrm{kV} / \mathrm{cm})^{-1}$.

Fig. 11. Distribution of the normal electric field $E_{n}$ for different $E_{\text {ext }}$ in the case of uniform charging with $\sigma_{0}$ equal to $-30 \mu \mathrm{C} / \mathrm{m}^{2}$ and the charge transfer given by $\sigma_{E 0}=-30 \mu \mathrm{C} / \mathrm{m}^{2}$ and $k_{E}=20\left(\mu \mathrm{C} / \mathrm{m}^{2}\right)$ $(\mathrm{kV} / \mathrm{cm})^{-1}$.

Fig. 12. Variation of $F_{a}$ with $E_{\text {ext }}$ on a partially charged particle with $k_{E}=20\left(\mu \mathrm{C} / \mathrm{m}^{2}\right)(\mathrm{kV} / \mathrm{cm})^{-1}$ and $\sigma_{E 0}=(a)-10$ and (b) $-30 \mu \mathrm{C} / \mathrm{m}^{2}$.

Fig. 13. Variation of $F_{a}$ with $E_{\text {ext }}$ on a partially charged particle with $\sigma_{E 0}=-30 \mu \mathrm{C} / \mathrm{m}^{2}$ and $k_{E}=$ (a) 10 or $(\mathrm{b}) 30\left(\mu \mathrm{C} / \mathrm{m}^{2}\right)(\mathrm{kV} / \mathrm{cm})^{-1}$. 


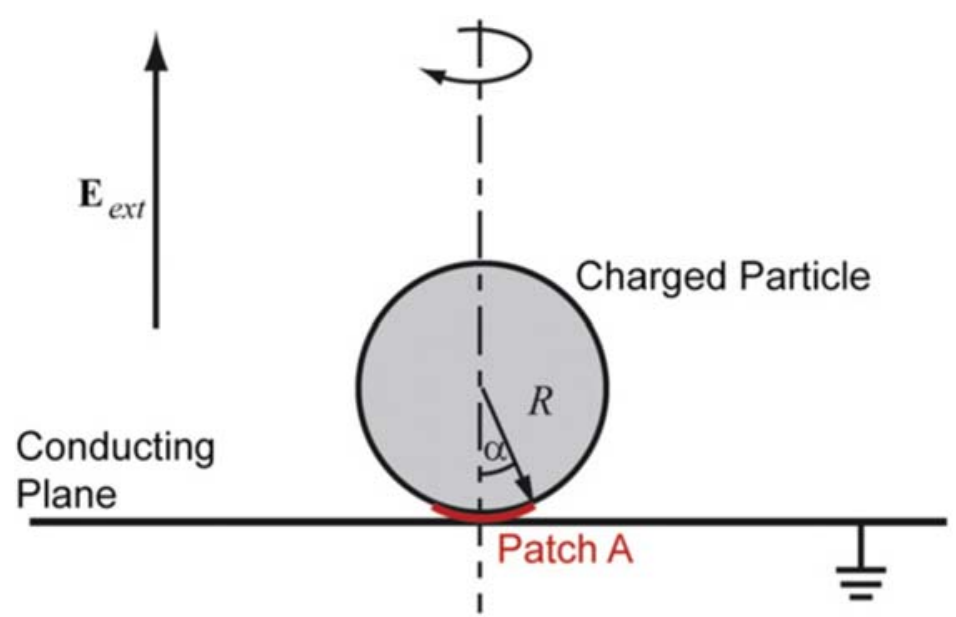

Fig. 1

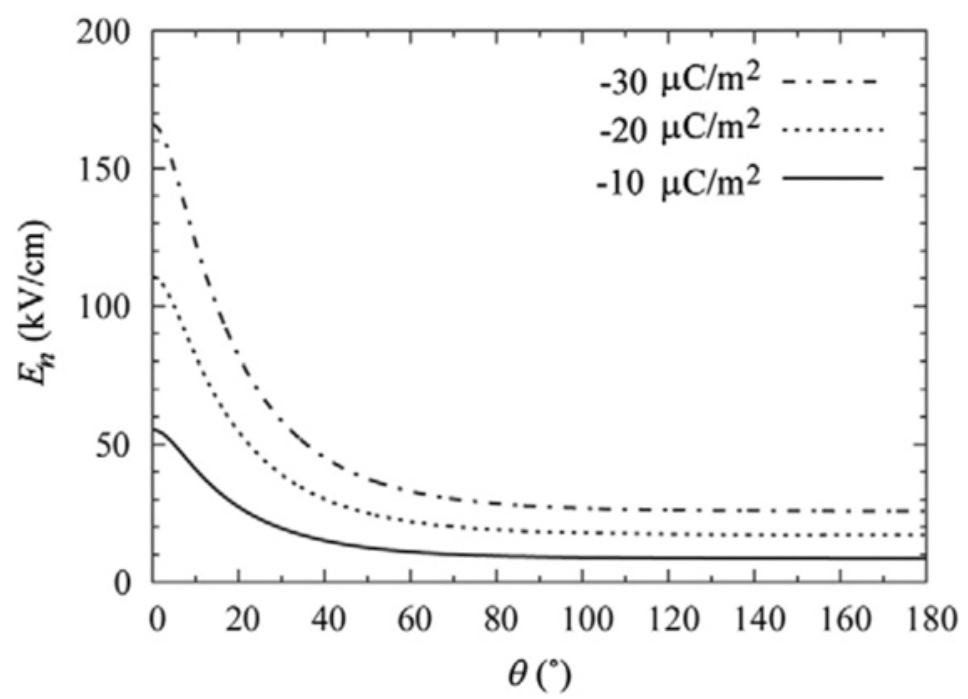

Fig. 2 


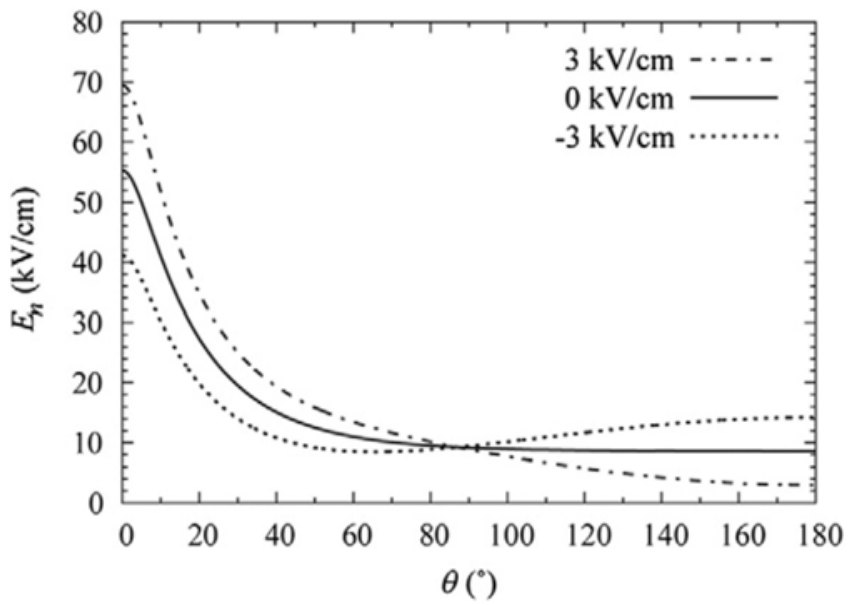

Fig. 3
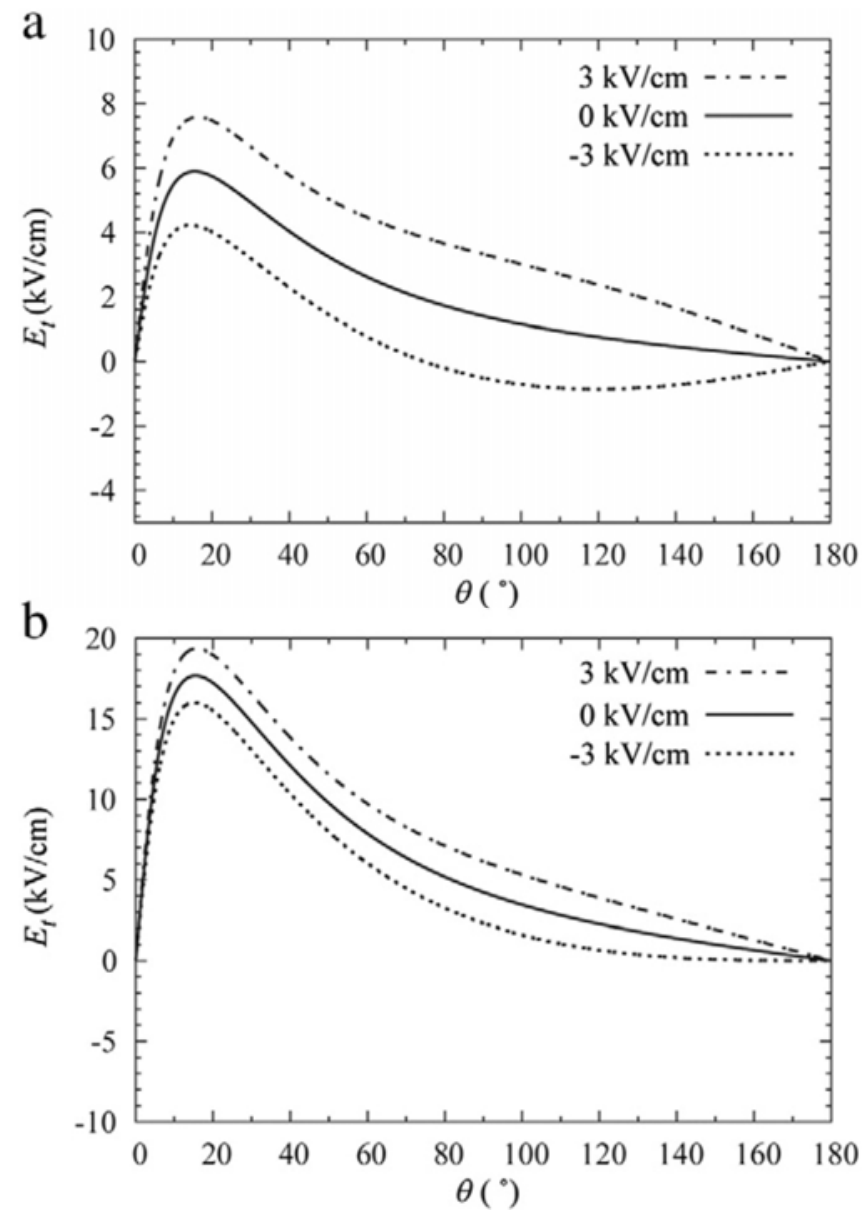

Fig. 4 


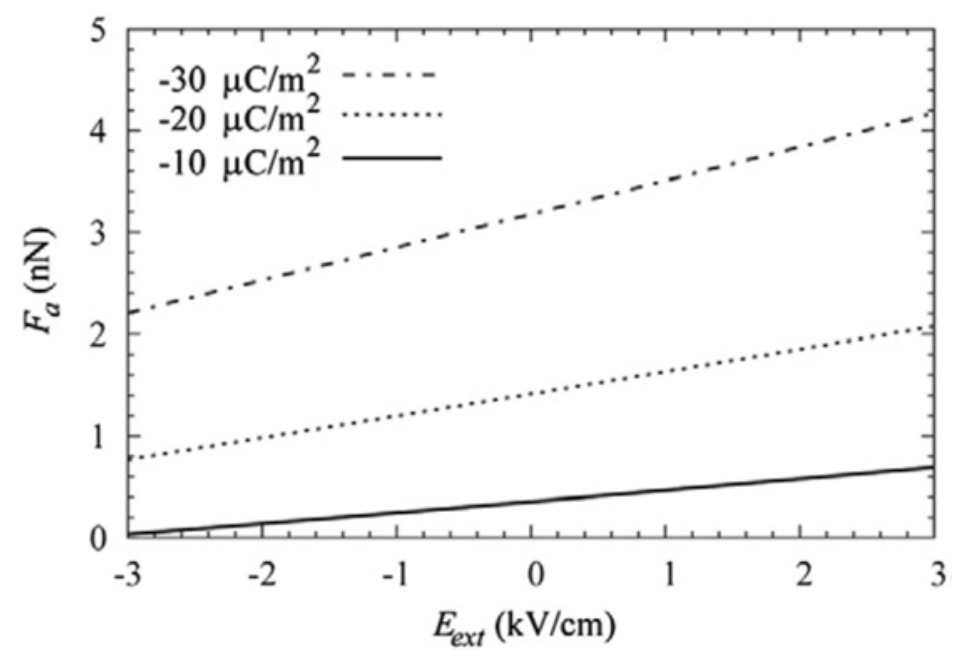

Fig. 5

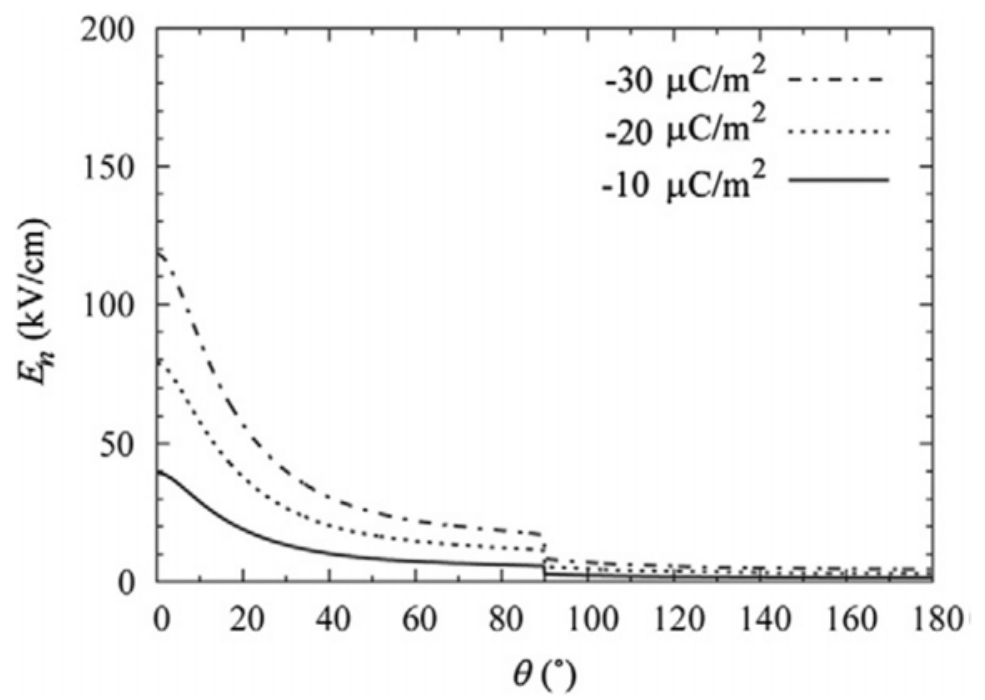

Fig. 6 

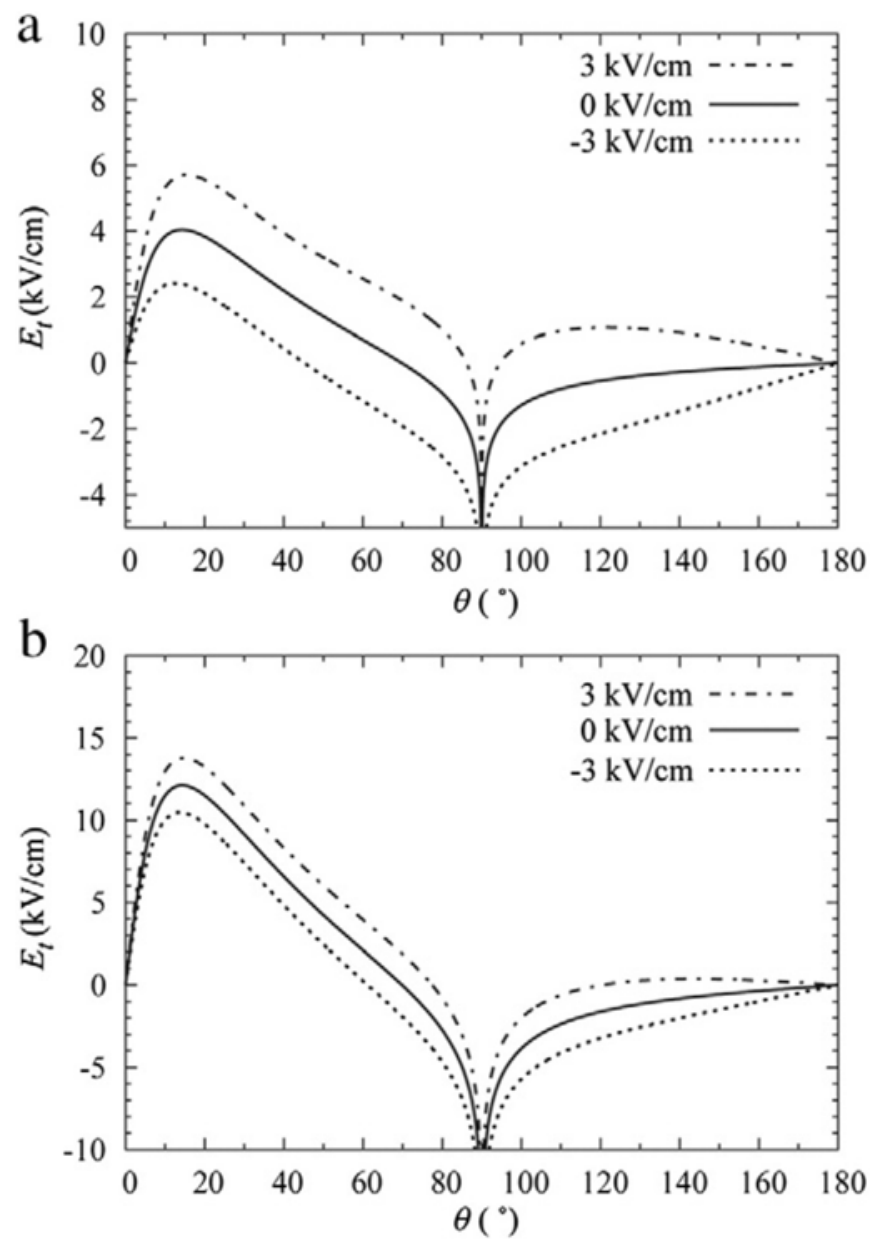

Fig. 7

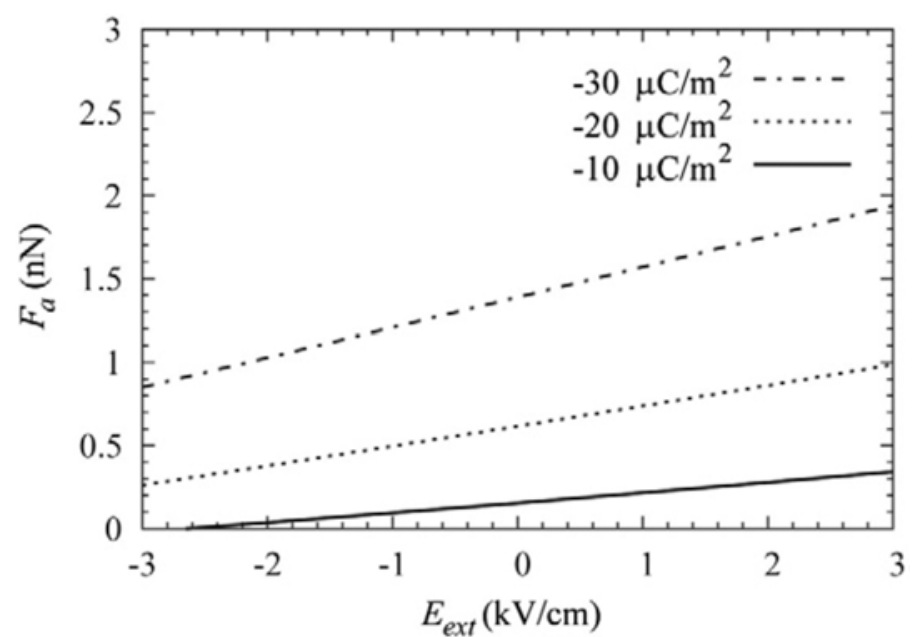

Fig. 8 

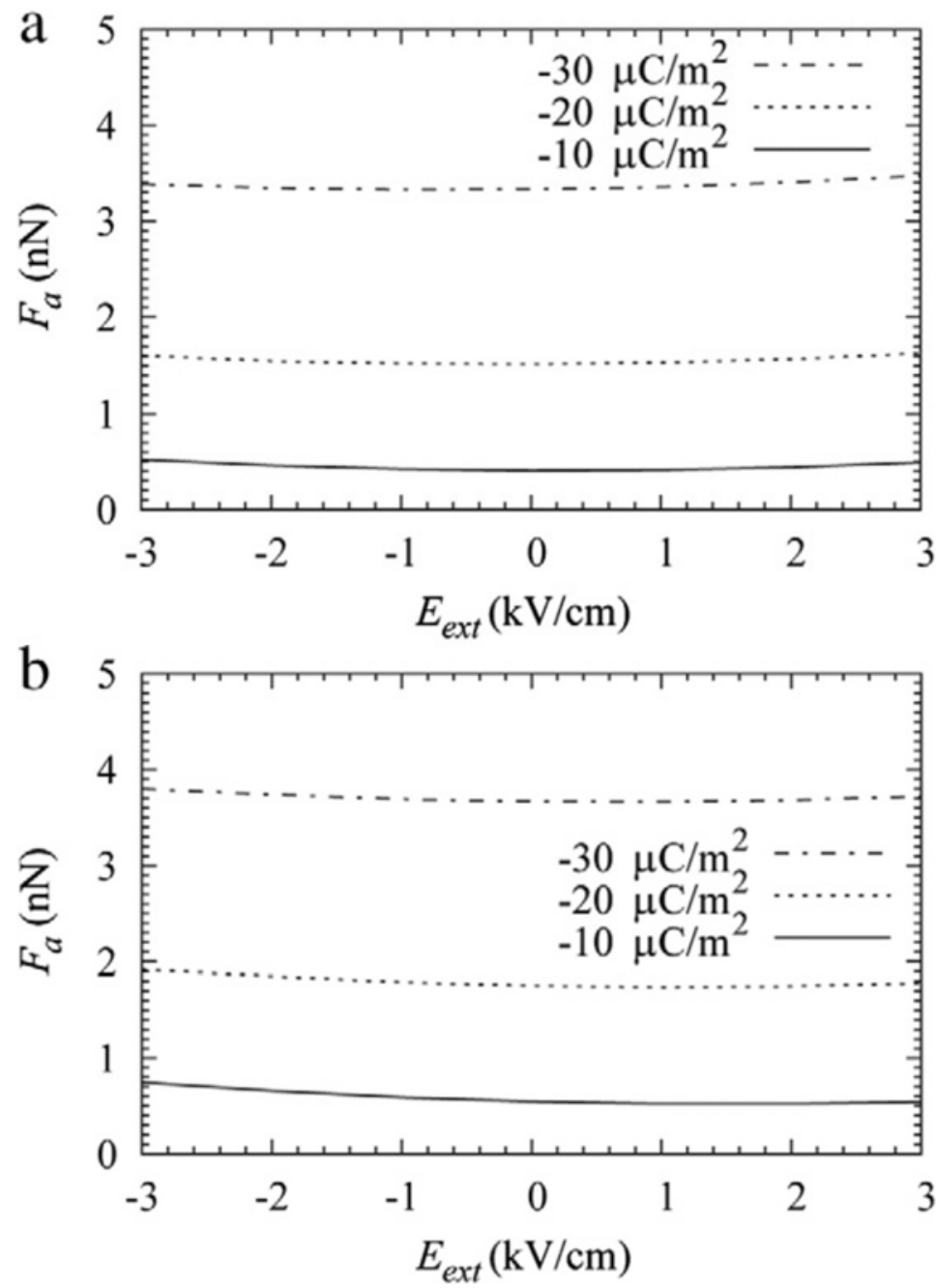

Fig. 9 

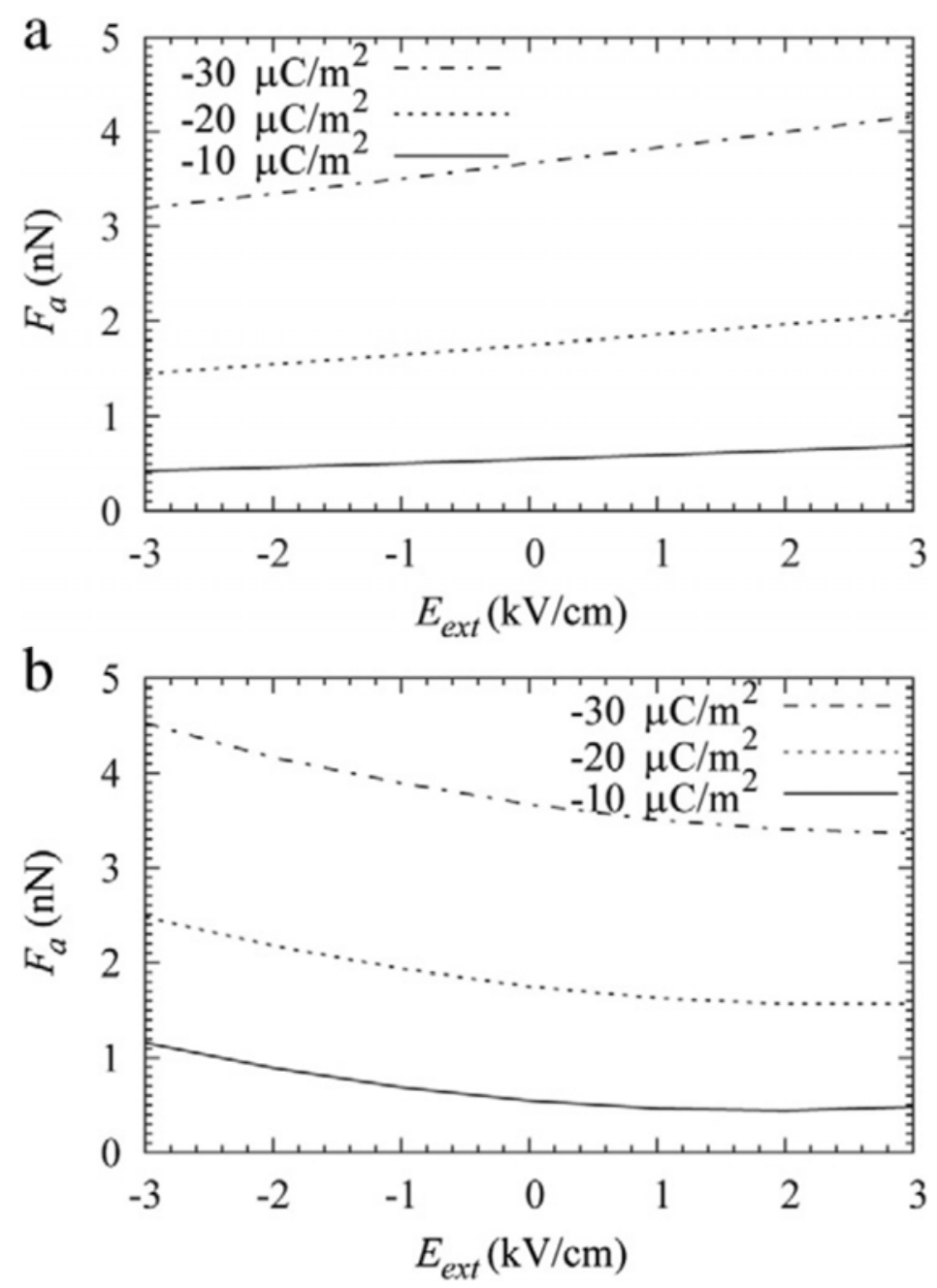

Fig. 10 


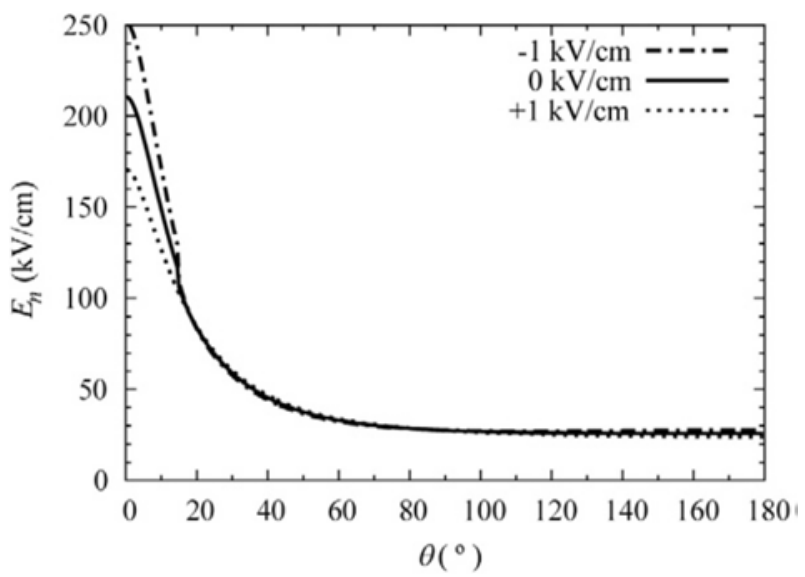

(b)

Fig. 11
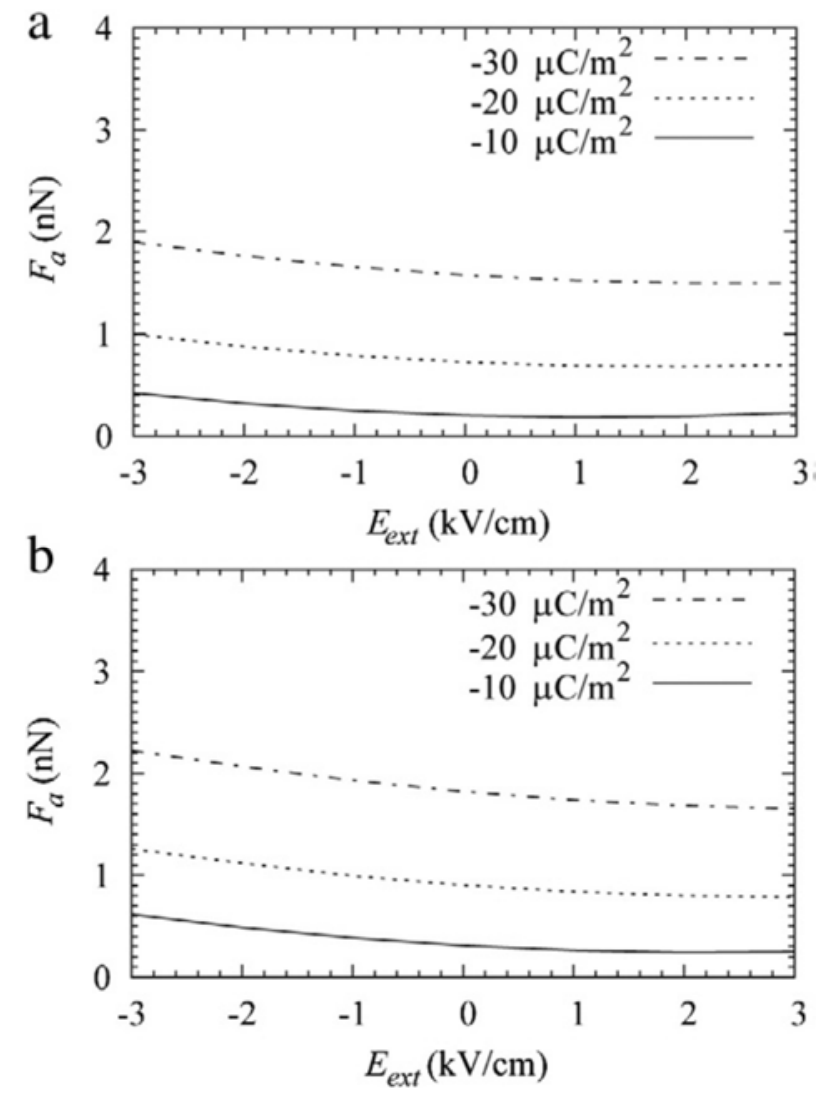

Fig. 12 

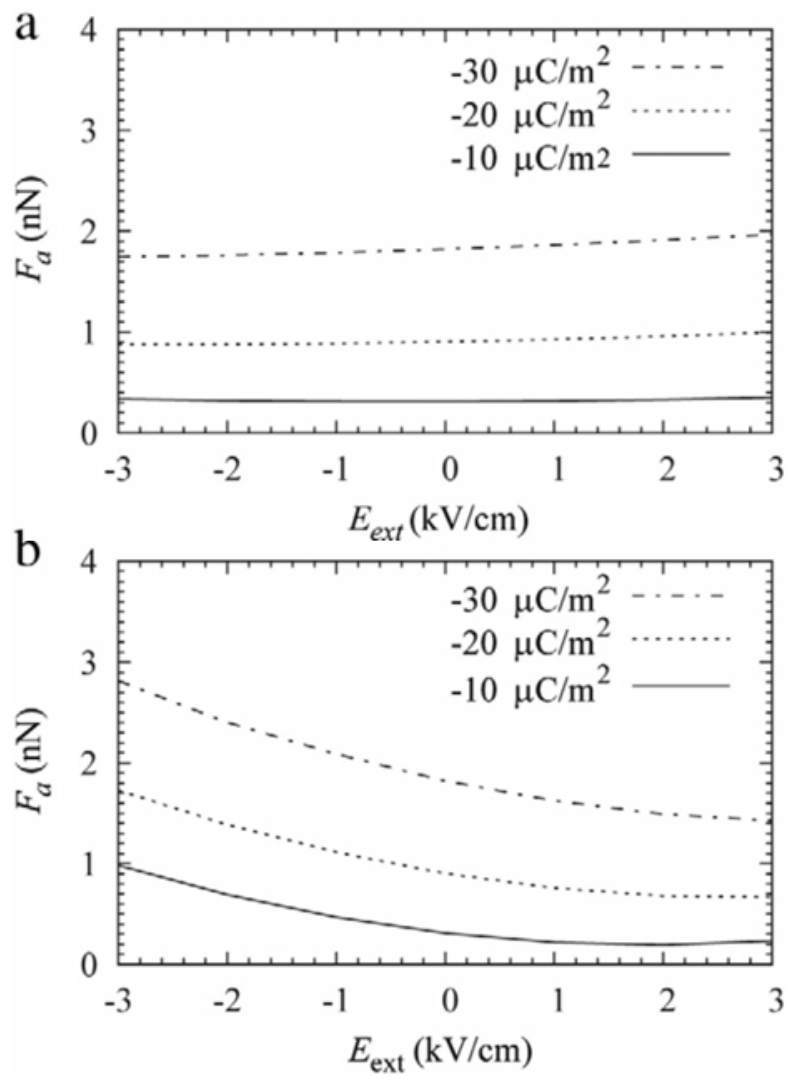

Fig. 13 